\title{
Platelet microparticle-mediated transfer of miR-939 to epithelial ovarian cancer cells promotes epithelial to mesenchymal transition
}

\author{
Meiling Tang ${ }^{1, *}$, Lu Jiang ${ }^{1, *}$, Yingying Lin ${ }^{2}$, Xiaoli Wu ${ }^{1}$, Kai Wang ${ }^{3}$, Qizhi He ${ }^{4}$, Xipeng \\ Wang $^{5}$ and Weiping Lí \\ ${ }^{1}$ Department of Gynecology, Shanghai First Maternity and Infant Hospital, Tongji University School of Medicine, Shanghai, China \\ ${ }^{2}$ Department of Neurosurgery, Renji Hospital, School of Medicine, Shanghai Jiao-Tong University, Shanghai, China \\ ${ }^{3}$ Central Laboratory, Shanghai First Maternity and Infant Hospital, Tongji University School of Medicine, Shanghai, China \\ ${ }^{4}$ Department of Pathology, Shanghai First Maternity and Infant Hospital, Tongji University School of Medicine, Shanghai, China \\ ${ }^{5}$ Department of Gynecology and Obstetrics, Xinhua Hospital, Affiliated with Shanghai Jiao Tong University, School of Medicine, \\ Shanghai, China \\ ${ }^{6}$ Department of Obstetrics and Gynecology, Renji Hospital Affiliated to Shanghai Jiao Tong University School of Medcine, \\ Shanghai, China \\ "These authors have contributed equally to this work \\ Correspondence to: Xipeng Wang, email: wangxipeng@xinhuamed.com.cn \\ Weiping Li, email: weipingli60@outlook.com
}

Keywords: EOC; PMPs; epithelial - mesenchymal transition (EMT); miR-939; secretary phospholipase A2 type IIA (sPLA 2 -Ila) Received: December 08, 2016

Accepted: August 26, 2017

Published: October 27, 2017

Copyright: Tang et al. This is an open-access article distributed under the terms of the Creative Commons Attribution License 3.0 (CC BY 3.0), which permits unrestricted use, distribution, and reproduction in any medium, provided the original author and source are credited.

\section{ABSTRACT}

Epithelial ovarian cancer (EOC) patients frequently suffer from thrombocytosis, which leads to a poor prognosis. However, the mechanism underlying platelet regulation of biological behavior in EOC remains unclear. The associations between clinicopathological characteristics and thrombocytosis in 171 EOC patients were studied, preoperative thrombocytosis was significantly associated with the stage, metastasis scope, level of preoperative CA125 and overall survival. When SKOV3 cells were cocultured with platelet microparticles (PMPs), the expression of molecules associated with epithelial-mesenchymal transition (EMT) was increased. The proliferation and migration of SKOV3 cells were also enhanced. Based on the miRNA microarray of the PMPs derived between thrombin-stimulating and apoptotic platelets, we demonstrated that over-expression or complete knockdown of miR-939 in the SKOV3 cells strengthened or weakened EMT. Secretory phospholipase A2 type IIA (sPLA ${ }_{2}$-IIa) has been shown to mediate PMPs intake by SKOV3 cells. The knockdown

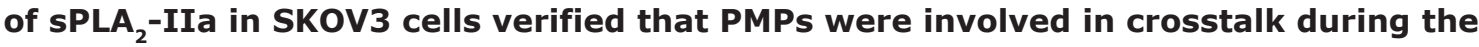
regulation of cancer cells by transferring miRNA. This study revealed an important role for PMPs in the crosstalk of platelets and cancer cells through miR-939 shedding

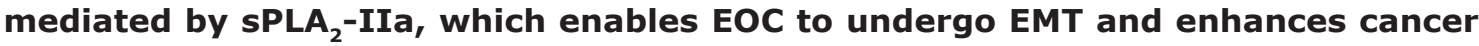
progression. Our findings pave the way for developing a novel therapeutic strategy for EOC targets such as PMPs, miR-939 or sPLA ${ }_{2}$-IIa.

\section{INTRODUCTION}

Epithelial ovarian cancer (EOC) accounts for $90 \%$ of all ovarian cancers and has become the fifth leading cause of cancer-related death among women [1]. Due to the lack of efficient screening programs, approximately $70 \%$ of patients are diagnosed at an advanced stage (FIGO stage III and IV), resulting in a $30 \%$ of 5 -year overall survival (OS) rate [2]. Some of the various clinicopathologic characteristics observed in advanced EOC include 
wide spread peritoneal metastases and thrombocytosis. However, the relationship between abundant platelets and tumor metastasis remains unclear.

The association between coagulation and cancer dates back to 1865 . Some EOC patients present with thrombocytosis as their initial symptom [3]. Clinical data indicate that paraneoplastic thrombocytosis is a prevalent phenomenon in patients with many types of solid tumors (gastrointestinal, lung, breast, or ovarian cancer) associated with cancer cell progression, such as angiogenesis, invasion, metastasis, poor survival, chemoresistance, and recurrence $[4,5]$. EOC treatments targeting platelets (anti-IL6 or thrombopoietin receptor therapy) could significantly inhibit the progression of EOC [2].

One of the major mechanisms involved in platelet-cancer cell interactions is tumor cell-induced platelet-aggregation (TCIPA) [6]. TCIPA leads to the formation of platelet cancer aggregates that adhere to the endothelium and may cause distant embolization of the microvasculature, which stimulates angiogenesis $[7,8]$. The secretory function of platelets also plays a very important role in stabilizing tumor vasculature and preventing intratumoral hemorrhages. Platelet-derived factors, including $\alpha$ granules and dense granules, could promote cancer cell proliferation and metastasis and maintain angiogenic microvessel integrity [9].

Most recently, interest in from 0.1 to $1.0 \mu \mathrm{m}$ PMPs secreted from activated platelets is increasing. Because PMPs contain platelet-derived bioactive molecules including miRNA, growth factors, and other signaling molecules. PMPs uptake induces functional changes in target cells. For example, PMP miRNAs can silence mRNA targets. Therefore, we compared the mRNA profiles of thrombin-stimulating platelet-derived PMPs against apoptotic platelet PMPs using a microarray assay to screen functional miRNA associated with EOC malignant behavior. In addition, secretory phospholipase A2 type IIA ( sPLA $_{2}$-IIa) is secreted by platelets and can induce PMPs internalized in neutrophils $[10,11]$. Thus, we hypothesized that SPLA $_{2}$-IIa is likely a key factor participating in the process of PMPs uptake by cancer cells.

In this study, we demonstrated that the selective uptake of PMPs by EOC could promote the initiation of EMT and ultimately support metastasis in ovarian cancer. To clarify the role of PMPs in EOC progression, we investigated the mechanism of PMPs containing miRNA engaging in crosstalk between platelets and EOC cells.

\section{RESULTS}

\section{EOC patients exhibited a high level of platelets}

The clinical demographic data are listed in Supplementary Table 1 . Of the 3 groups with ovarian tumors, the platelets in the EOC patients were elevated significantly $\left(183.3 \times 10^{9} / \mathrm{L}\right.$ in benign, $206.9 \times 10^{9} / \mathrm{L}$ in borderline, $248.0 \times 10^{9} / \mathrm{L}$ in EOC, p $<0.001$, Supplementary Table 1, Figure 1A) according to blood cell analyses. With threshold platelet counts of $350 \times$ $10^{9} / \mathrm{L}$ and $400 \times 10^{9} / \mathrm{L}$, the rate of thrombocytosis was $11.7 \%(20 / 171)$ and $7 \%(12 / 171)$ in the EOC patients in the Chinese population (Supplementary Table 1). If the general criterion of thrombocytosis of 400 or $450 \times 10^{9}$ /L was selected, the rate of thrombocytosis would be relatively low. Thus, $350 \times 10^{9} / \mathrm{L}$ was selected for this study. Stage III and stage IV EOC patients exhibited significantly higher levels of preoperative platelets than stage I and stage II EOC patients (Figure 1B).

\section{High levels of platelets predicted an advanced stage, optimal surgery and overall survival}

As shown in Supplementary Table 2, no significant differences in age, hemoglobin, lymphocyte count, prothrombin time(PT), tumor markers after the third cycle of chemotherapy, or the preoperative platelet to postoperative platelet ratio (defined as the preoperative platelet count divided by the platelet count after three cycles of primary adjuvant chemotherapy) were noted among the 171 EOC cases with thrombocytosis and nonthrombocytosis. With respect to the clinical demographic data, signs of ascites $(\mathrm{P}=0.029)$ were remarkable in the EOC patients with thrombocytosis, which suggests that more advanced clinical symptoms were associated with thrombocytosis. In surgical pathology, $60 \%$ of the EOC patients with thrombocytosis exhibited a residual tumor size greater than $1 \mathrm{~cm}$ after the debulking surgery compared with $29.1 \%$ of the EOC patients with non-thrombocytosis. This difference was statistically significant $(\mathrm{p}=0.01)$ (Figure $1 \mathrm{C})$. Thus, more cases of EOC with thrombocytosis were observed in the advanced stage (FIGO III and IV) compared with the of EOC patients without thrombocytosis $(\mathrm{P}=0.029)$ (Figure 1C). With respect to organ involvement during the operation, more organs, including the omentum $(p=0.006)$, diaphragmatic surface $(\mathrm{p}=0.018)$ and peritoneum $(\mathrm{p}=$ 0.016 ), exhibited a higher incidence of involvement in the EOC patients with thrombocytosis compared with patients without thrombocytosis. Besides, the level of preoperative CA125 was positively correlated with preoperative platelet $(\mathrm{p}<0.05)$ (Figure 1D).

We also studied the preoperative platelet count at different levels to determine the most optimal cutoff value to predict an optimal surgery at $232.2 \times 10^{9} / \mathrm{L}$, advanced disease stage at $208.5 \times 10^{9} / \mathrm{L}$ and overall survival at $327.5 \times 10^{9} / \mathrm{L}$ (Supplementary Table 3 ). From the table, a preoperative platelet count of $232.2 \times 10^{9} / \mathrm{L}$ yielded the most optimal predictive value to predict a suboptimal surgery. The AUC for determining a suboptimal surgery was 0.62 (95\% confidence interval (CI), 0.53 to 0.71 ), 
whereas the sensitivity, specificity, positive predictive value (PPV), and negative predictive value (NPV) were $62.5 \%, 57.39 \%, 42.61 \%$, and $37.5 \%$, respectively. A preoperative platelet count of $208.5 \times 10^{9} / \mathrm{L}$ yielded the best predictive values for advanced-stage disease. The AUC was 0.62 (95\% CI, 0.54 to 0.71$)$, whereas the sensitivity, specificity, PPV and NPV were $73.17 \%$, $45.24 \%, 54.7 \%$ and $26.83 \%$, respectively. To determine overall survival, a preoperative platelet count of $327.5 \times$ $10 \% / \mathrm{L}$ exhibited an AUC of 0.80 (95\% CI, 0.66 to 0.95$)$, a sensitivity of $64.29 \%$, specificity of $93.75 \%$, PPV of $6.25 \%$ and NPV of $35.71 \%$. All the EOC patients were followed for a median of 30 months (3-55 months). When using the different preoperative platelet cutoff values of $300 \times 10^{9} / \mathrm{L}, 350 \times 10^{9} / \mathrm{L}$ and $400 \times 10^{9} / \mathrm{L}$, the EOC patients with thrombocytosis exhibited a significantly shorter overall survival time (median: 37.5 . months; 36.4 months; 32.1 months, respectively) compared with those with platelet counts less than $300 \times 10^{9} / \mathrm{L}, 350 \times 10^{9} / \mathrm{L}$ and $400 \times 10^{9} / \mathrm{L}$ (median: 52.1 months; 50.8 months; 51.0 months, respectively; $\mathrm{p}<0.0001$ ) (Figure $2 \mathrm{~A}-2 \mathrm{C}$ ). Thus, high platelet levels could predict an advanced stage, optimal surgery and overall survival.

\section{PMPs induce proliferation and migration of ovarian cancer cells}

The collected platelets from the blood of healthy human donors that were stimulated with thrombin to obtain PMPs were analyzed. The quantity of protein was quantified using the BCA method [12]. Using a scanning electron microscope (SEM) to visualize isolated PMPs and determine their structure, the size of PMPs probably ranges from $0.05 \mu \mathrm{m}$ to $0.8 \mu \mathrm{m}$ (Figure $3 \mathrm{~A}$ ). To determine
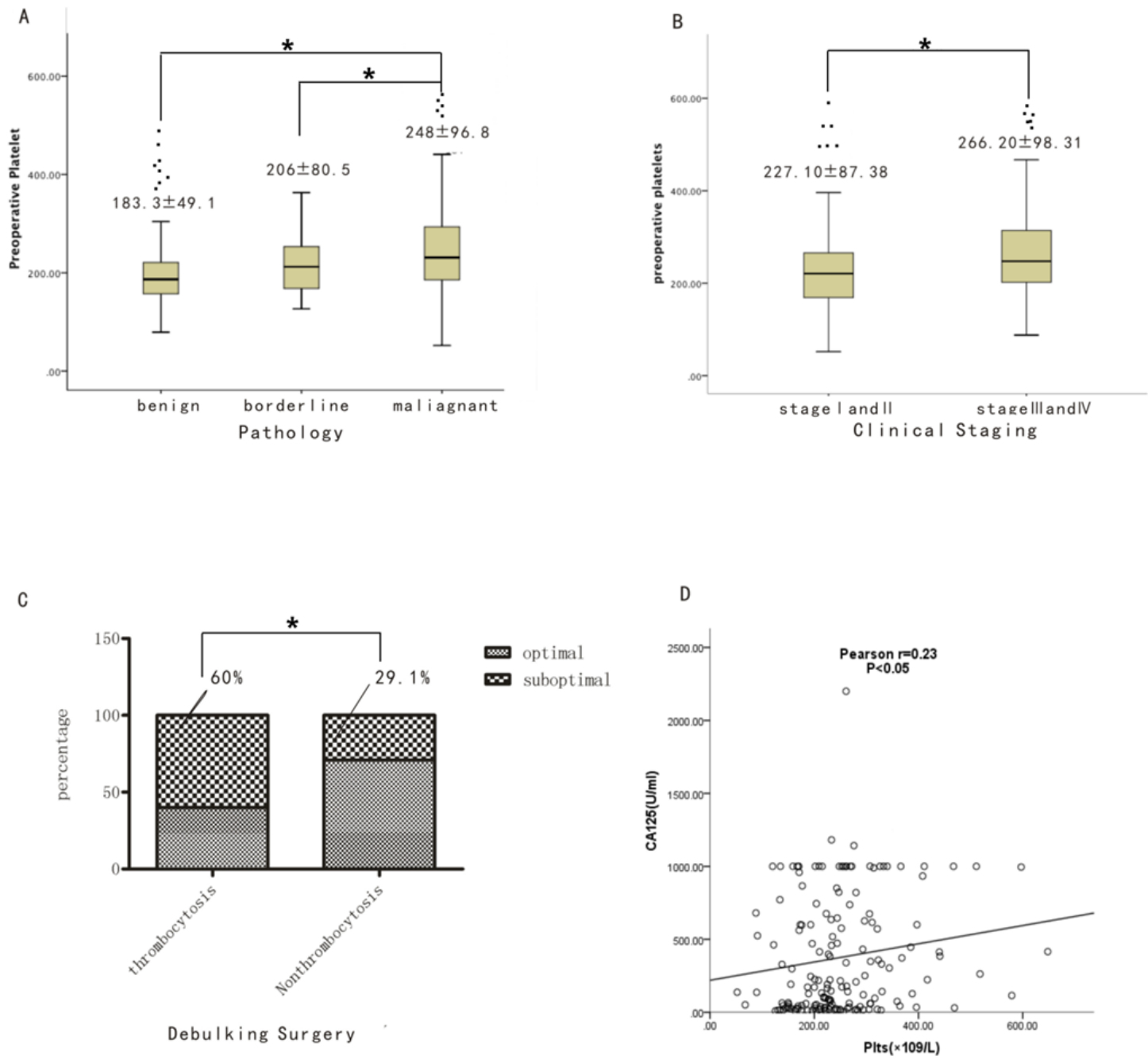

Figure 1: Comparison of EOC patients with thrombocytosis and non-thrombocytosis. (A) Comparison of preoperative platelet count among benign epithelial ovarian tumor, borderline ovarian tumor and EOC (All $\mathrm{P}<0.05$ ). (B) Comparison of preoperative platelets count between EOC at early stage (I and II) and advanced stage (III and IV) $(\mathrm{P}<0.05)$. (C) Percentage of optimal debunking surgery between EOC with thrombocytosis and EOC without it $(\mathrm{P}<0.05)$. (D) Linear regression analysis of CA125 levels with preoperative platelets in $\mathrm{EOC}(\mathrm{P}<0.05)$. 
A

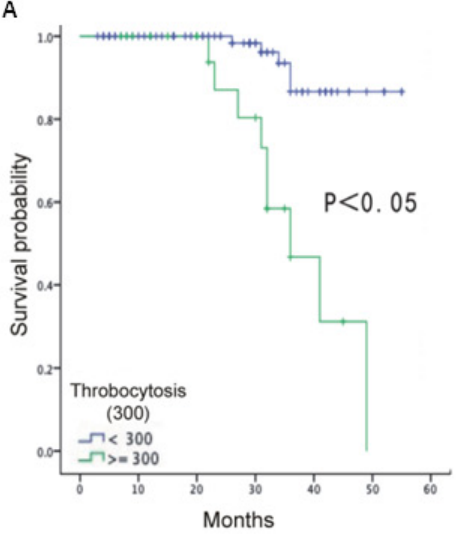

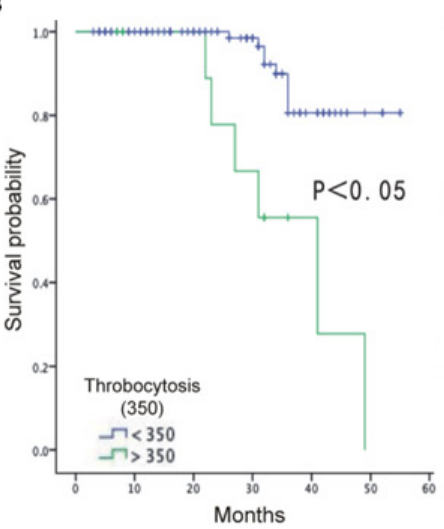

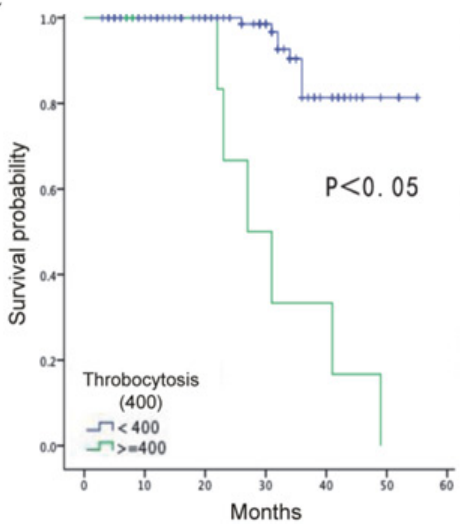

Figure 2: Overall survival of EOC patients based on definition of thrombocytosis at $300 \times 10^{9} / \mathrm{L}$ (A), $350 \times 10^{9} / \mathrm{L}$ (B) and $400 \times 10^{9} / \mathrm{L}(\mathbf{C})$.

A

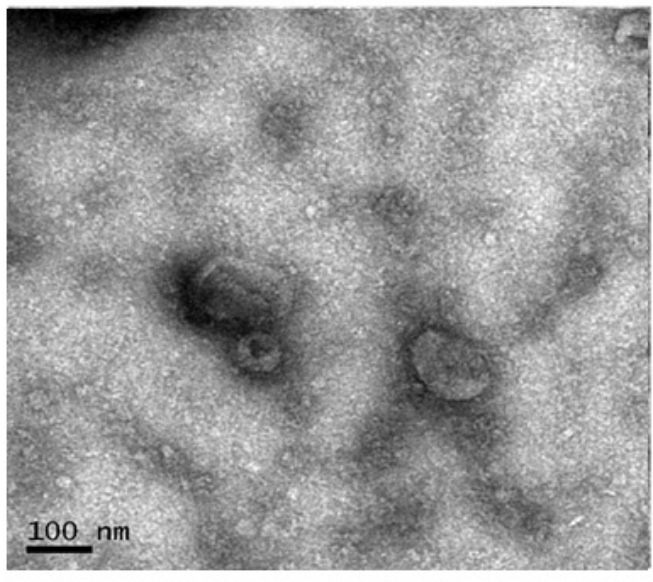

B

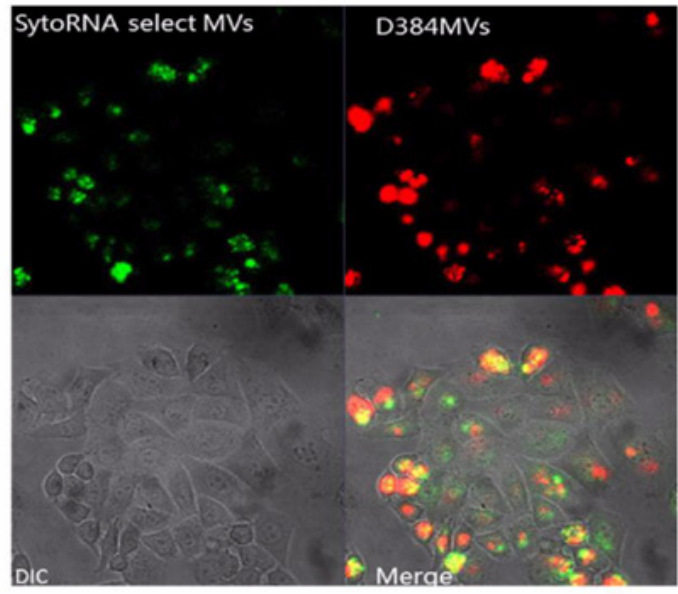

C
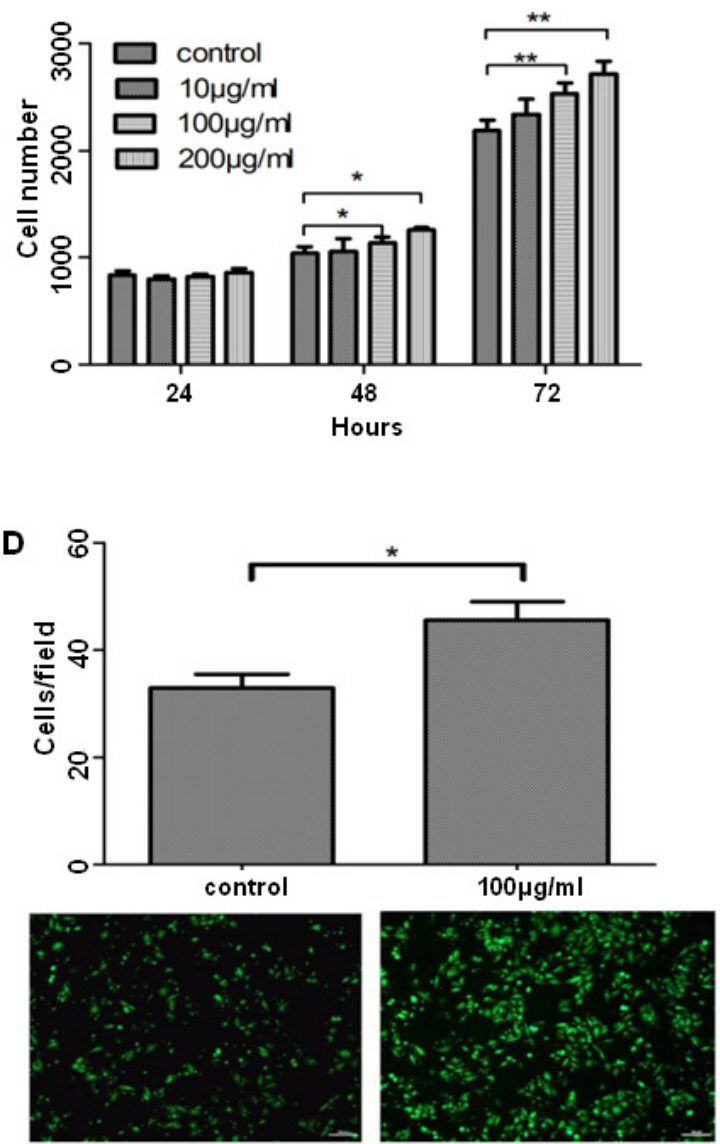

Figure 3: PMPs induce proliferation and migration of ovarian cancer cells. (A) Scanning electron microscope (SEM) of PMPs. The size of PMPs is probably ranging from $0.05 \mu \mathrm{m}$ to $0.8 \mu \mathrm{m}$. (B) Confocal images of PMPs uptake by SKOV 3 cells. PMPs stained with D384, a phospholipids membrane dye (red stain; top left panel) and SytoRNA Select, an RNA-select stain (green stain; bottom left panel). PMPs $(100 \mu \mathrm{g} / \mathrm{ml})$ are incubated with unstained naive recipient cells for 12 hours. (C) The proliferation rate of SKOV3 cells increase

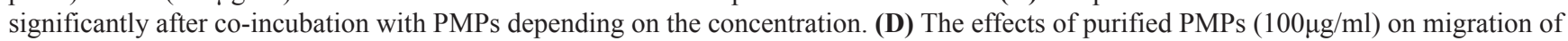
SKOV3 cells are determined by using 24 well Transwell Permeable Supports with $8 \mu \mathrm{M}$ pores. Values of relative fluorescence are shown. $\mathrm{n}=3 .{ }^{*} \mathrm{p}<0.05$ compared to control. ${ }^{* *} \mathrm{p}<0.01$ compared to control. Scale bar, $100 \mu \mathrm{m}$. 
the mechanism of PMPs uptake by SKOV3 cells, we cocultured the PMPs and SKOV3 cells and observed the uptake process using confocal microscopy. The fluorescent PMPs and transferred RNA were apparent in the recipient cell membranes and cytoplasm by the appearance of the red dye and green stain, respectively (Figure 3B). SKOV3 cells treated with concentrations of PMPs ranging from 10 to $200 \mu \mathrm{g} / \mathrm{ml}$ were cultured for 3 days to study their proliferation. The results revealed that the growth of the cells increased significantly over time and with higher PMPs concentrations (Figure 3C). We also observed that the PMPs promoted the migration of the SKOV3 cells by approximately $15 \%$ (Figure 3D). Consequently, the PMPs were internalized by endocytosis and promoted the proliferation and migration of the cancer cells.

\section{PMPs enhance the metastasis of EOC by inducing EMT}

PMPs can promote tumor metastasis. However, the mechanism remains unclear. The cancer cells co-cultured with $100 \mu \mathrm{g} / \mathrm{ml}$ PMPs were analyzed for mesenchymal markers and transcription factors involved in EMT. E-cadherin was down-regulated, whereas N-cadherin, vimentin, snail, fibronectin, MMP-2, MMP-9, and ZEB2 were up-regulated. The results indicate that the ovarian cancer cells with PMPs tended to spindle and exhibit a fibroblastic-type phenotype (Figure 4B). The expression of E-cadherin obviously decreased at an early stage, and increased expression of N-cadherin followed (Figure 4B). Next, we co-cultured the cells for 4 days and analyzed other factors associated with EMT. Twist, Slug, ZEB2, ZEB1, snail, vimentin, fibronectin, interleukin (IL)-8, and IL-6 also increased. The RNA and protein levels indicated that EMT had occurred in the cancer cells (Figure 4C and 4D). Thus, PMPs could induce EMT in ovarian cancer cells.

\section{miR-939 promotes the epithelial-mesenchymal transition in ovarian cancer}

Next, we used a significance microarray analysis and analyzed the miRNA differences between the two
A

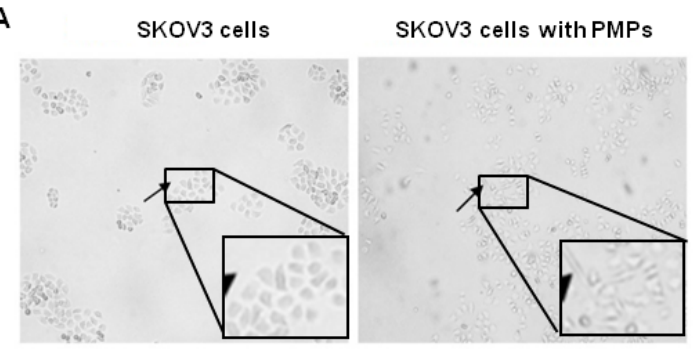

C

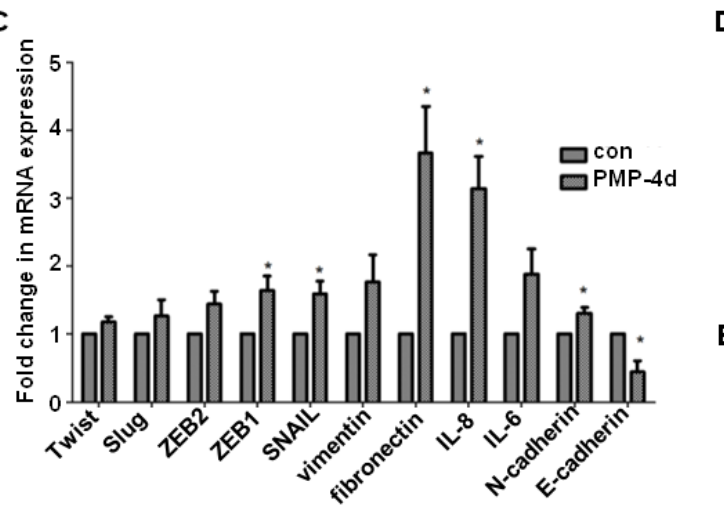

EMT markers

\section{B}
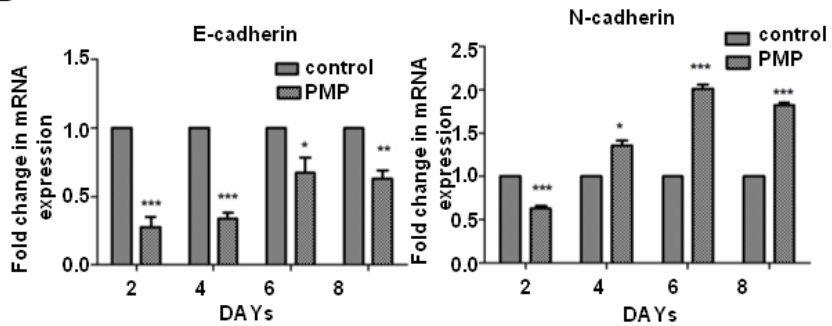

D

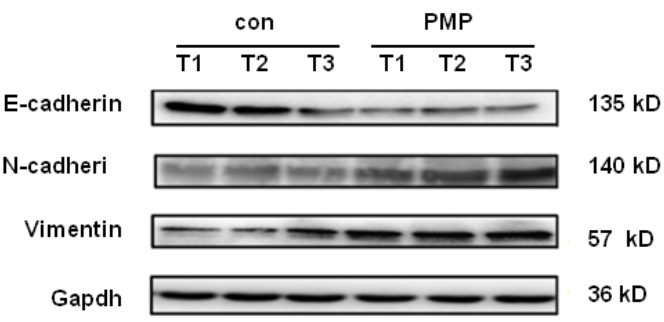

$\mathbf{E}$

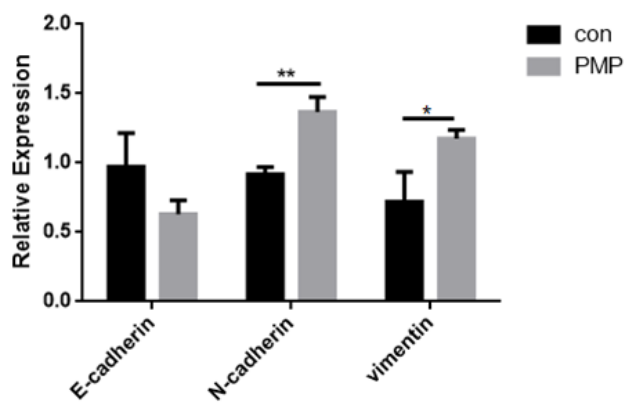

Figure 4: PMPs induce EMT of Ovarian cancer cells. (A) SKOV3 cells are stimulated for 48 hours with PMPs (100 $\mu \mathrm{g} / \mathrm{ml})$. Photomicrographs of SKOV3 cells show a rounded epithelial cell shape (left panel) and a fibroblastic-type phenotype when cocultured with PMPs (right panel); original magnification, $\times 40$. (B) Real-time PCR analysis of E-cadherin and N-cadherin at different time. (C) Real-time PCR analysis of transcription factors and EMT-specific genes in SKOV3 cells co-cultured with PMPs for 4 days. The relative mRNA levels were normalized to $\beta$-actin. (D, E) Western blot analysis of EMT-specific proteins in SKOV3 cells co-cultured with PMPs for 8 days. Gapdh served as the loading control. T1, T2 and T3 stand for the first, second and third time. $\mathrm{n}=3$. ${ }^{*} \mathrm{P}<0.05$. ${ }^{* *} \mathrm{P}<0.01,{ }^{* * *} \mathrm{P}<0.001$. 
types of PMPs from the platelets stimulated by thrombin or apoptosis (Figure 5A). After consulting the literature, we found that in addition to miR-939, other miRNAs with obvious differences have no significant correlation with tumor. Therefore, we hypothesized that miR-939 in PMPs would release and activate cancer cells after being absorbed by ovarian cancer cells, thus promoting the progression of ovarian cancer. In the SKOV3 cells transfected with miR-939 mimics, miR-939 inhibitor or the respective controls, the expression of miR-939 was verified by qRTPCR (Figure 5B and 5E). The expression of mRNAs translated after 24 hours is related to EMT-specific genes changes. The protein and mRNA levels of E-cadherin decreased, whereas vimentin, fibronectin, ZEB2 and slug expression increased (Figure 5C and 5D). The miR939 knockdown in the cancer cells resulted in epithelial cell features. The expression of E-cadherin was highexpressed, whereas vimentin expression decreased as time progressed. The related proteins exhibited more obvious changes (Figure 5E and 5F). In addition, we also found miR-939 could improve the proliferation and migration of ovarian cancer cells (Supplementary Figure 2).
Therefore, miR-939 might be the key element that mediates the interaction between platelets and cancer cells, thereby promoting metastasis though EMT.

\section{SPLA - -IIA induces a combination of PMPs and ovarian cancer cells}

PMPs can be internalized by neutrophils through sPLA $_{2}$-IIA [10]. We hypothesized that $\mathrm{sPLA}_{2}$-IIa induces the combination of PMPs and ovarian cancer cells. SPLA IIA (Figure 6A) was knocked down in the SKOV3 cells via siRNA. The resulting SKOV3 cells were co-cultured with PMPs for 4 or 8 days, and mRNA and protein levels were assessed at both time points. The results revealed that E-cadherin was increased when PLA 2 -IIA was knocked down in the SKOV3 cells. In the co-culture of PMPs and SPLA $_{2}$-IIA knockdown SKOV3 cells, E-cadherin expression increased. However, vimentin expression decreased (Figure 6B and 6C). Thus, sPLA 2 -IIA could promote the process of internalization of PMPs by ovarian cancer cells.
A

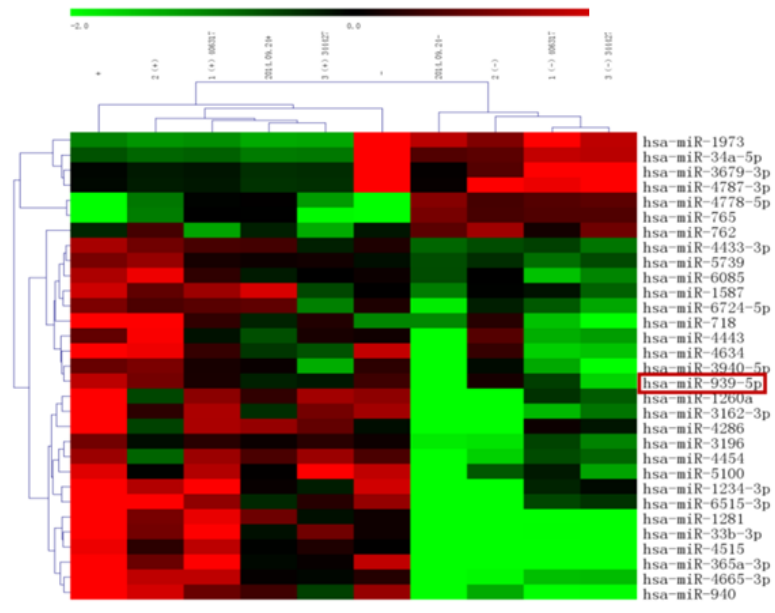

$E$

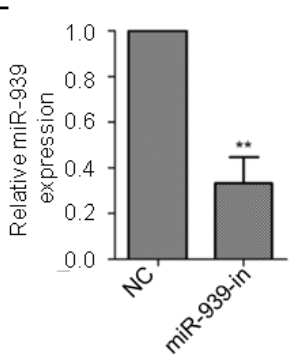

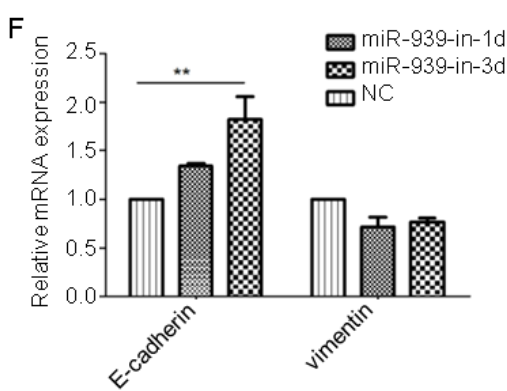

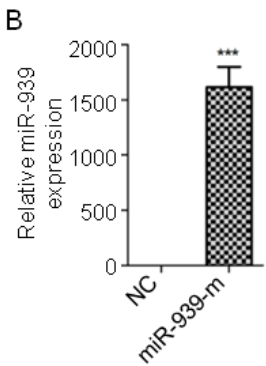

C

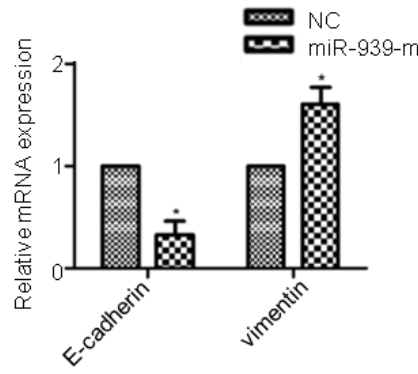

D

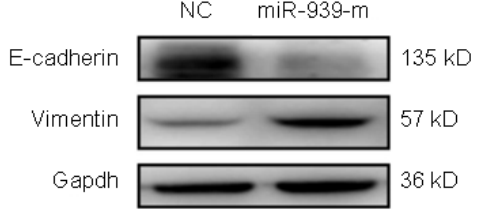

G

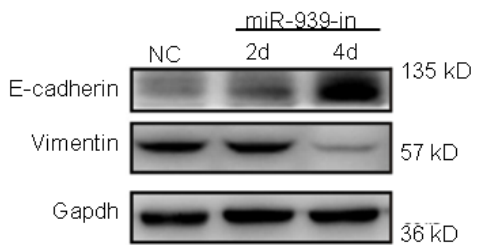

Figure 5: miR-939 promotes EMT in ovarian cancer. (A) Heatmap of the altered miRNA expression in PMPs from platelets stimulated by thrombin or apoptosis. Validation of miR-939 expression levels after transfecting miR-939 mimics(miR-939-m) (B) or inhibitor (miR-939-in) (E) by PCR analysis. Real-time PCR analysis of the EMT-specific genes in SKOV3 cells transfected miR-939 mimics (C) or miR-939 inhibitor after 1(miR-939-in-1d) or 3(miR-939-in-3d) days (F). The relative mRNA levels were normalized to $\beta$-actin. Western blot analysis of E-cadherin and vimentin protein in SKOV3 cells transfected miR-939 mimics (D) or inhibitor (G). Gapdh served as the loading control. NC means normal control. $\mathrm{n}=3$. ${ }^{*} \mathrm{P}<0.05$. 


\section{DISCUSSION}

Although platelet and ovarian cancer have been studied for many years, our recent study analyzed novel features of their interaction. First, using a large amount of clinical data, we revealed the clinical significance of preoperative platelet aggregates in the progression of EOC. Second, we were the first to illuminate that PMPs induce the EMT of ovarian cancer by acting as the carrier in platelet-tumor cell crosstalk. Third, to date, no studies have demonstrated that miR-939 is associated with EMT. Fourth, we found that sPLA $\mathrm{PL}_{2}$-IIA could promote PMPs uptake by cancer cells, which is not often studied.

The prevalence of thrombocytosis in patients with ovarian cancer ranges from $22.3 \%$ to $68 \%$ [4, 13-15]. Generally, the cutoff value of thrombocytosis associated with cancer was as a platelet count at least $400 \times 109 / \mathrm{L}$ or $450 \times 10^{9} / \mathrm{L}$ in other studies. Only $7.0 \%$ of patients exhibited platelet counts greater than $400^{*} 109 / \mathrm{L}$ in this study. Another published study in China by Qiu, J et al. [16] found that 7.4\% (10/136) of EOC patients had thrombocytosis with platelet count $>400 \times 10^{9} / \mathrm{L}$, which was similar to our results. However, one study from Korean suggested that the incidence of thrombocytosis in EOC was $36.4 \%$ if more than $400 \times 10^{9} / \mathrm{L}$ was selected as the cutoff [17]. The authors suggested that the incidence of thrombocytosis varies among population from different Asian countries. In addition, in our study using the ROC curve, preoperative platelet count of $327.5^{*} 10^{9} / \mathrm{L}$, which was less than $350 \times 10^{9} / \mathrm{L}$, yielded the most optimal predictive value to predict overall survival. Therefore, thrombocytosis was defined as a platelet count great than $350 \times 10^{9} / \mathrm{L}$, and the incidence of thrombocytosis with EOC was $11.7 \%$ in this study.

Thrombocytosis has been consistently shown to be associated with advanced-stage and higher grade cancers, more frequent lymph node metastases, greater volumes of ascites, and reduced overall survival $[15,18,19]$. Some of our results were consistent with previous studies. In this study, the presence of thrombocytosis suggested that the platelet count in EOC was positively related to the CA125 level $(p=0.049)$, larger postoperative residue tumor volumes, more ascites formation, more organ involvement, surgical staging and poorer survival. Thus,
A
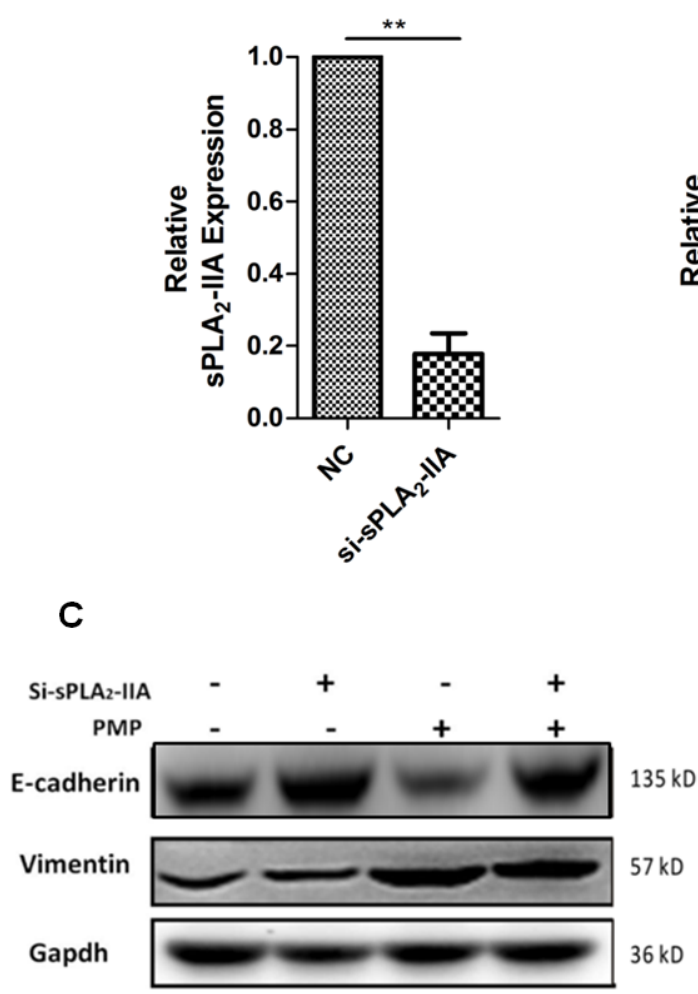

B

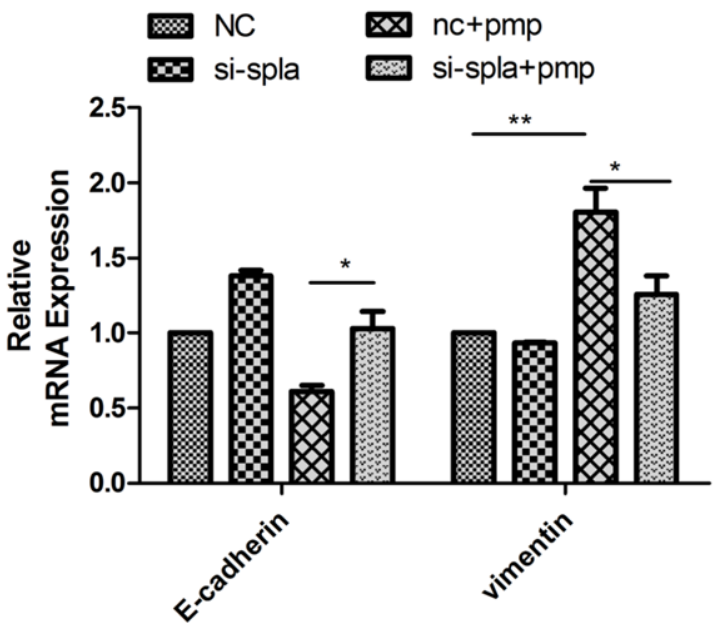

D

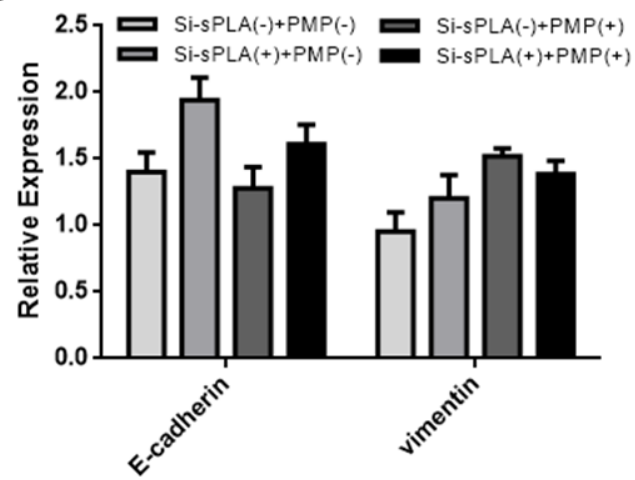

Figure 6: sPLA2-IIA induces the internalization of PMPs by ovarian cancer cells. (A) Validation of sPLA2-IIA expression levels after transfection by PCR analysis (B-C) Knockdown sPLA2-IIA in SKOV3 by siRNA, then co-cultured with PMPs for 4 days. Realtime PCR analysis of the change of EMT-specific genes in SKOV3 cells (B). The relative mRNA levels were normalized to $\beta$-actin. Western blot analysis of E-cadherin and vimentin protein in SKOV3 cells (C, D). Gapdh serves as the loading control. $\mathrm{n}=3$. 
anti-thrombosis treatments, including heparin [20-24] and anti-interleukin 6 antibody [4], in cancer patients could prolong survival by interfering with the crosstalk between tumors and platelets.

PMPs were regarded as inert "platelet dusts" with a potential role in the platelet-cancer loop. PMPs are the most abundant microparticles in the blood, constituting the majority $(>80 \%)$ of the pool of MPs [25]. PMPs transport miRNAs, growth factors and other bioactive molecules and participate in forming platelets [12, 18]. PMPs are also involved in the cascade of cellular injury and vascular dysfunctions underlying thrombosis, especially in cancer [26]. PMPs promote invasion of prostate cancer cells by up-regulating MMP-2 [27]. PMPs also promote proliferation, differentiation, and survival of neuronal cells, indicating a role in treating brain injuries. Probable mechanisms for this activity likely involve the pAKT and protein kinase RNA-like endoplasmic reticulum kinase (pERK) pathways [28]. As previously mentioned, we also found that PMPs promote ovarian cancer cell proliferation and migration and the expression of EMT-specific genes and promote EMT in ovarian cancer. However, the dynamic changes and special markers of tumor-associated PMPs in the tumor microenvironment, blood, and other regions, such as urine, have not been thoroughly explored. Understanding their mechanism has great value in early clinical screening and diagnosis.

Our microRNA microassay of PMPs derived from platelets stimulated by thrombin or naturally by apoptosis revealed a significant difference in miR-939. miR-939 is up-regulated in cancers, such as lung adenocarcinoma [29, 30] and high-risk neuroblastoma [31]. miR-939 regulates a large number of genes, such as WNT1, NTSR1, POLK, WWOX and SPN, that are related to lung cancer [32]. In ovarian cancer, miR-939 dramatically promoted ES-2 cell proliferation by suppressing APC2 expression [30, 33]. Given the results from our study, miR-939 may be the key factor in PMPs that induces the EMT. As expected, miR-939 levels were high in the PMPs. miR-939 downregulated E-cadherin and up-regulated vimentin. Also miR-939 could stimulate the proliferation and migration of ovarian cancer cells. These results indicate that miR939 could induce EMT and promote tumor progression, which is a novel finding. About its mechanism, we found that miR-939 promoted EMT of ovarian cancer cell with E-cadherin down-regulated. And there is a predicted consequential pairing of target region between miR-939 and the 3'UTR of E-cadherin (position 802808). Moreover, miR-939 has several potential target regions with the 3 'UTR regions of claudin (e.g. position 71-77). Based on these target region analysis, miR-939 might promote EMT via down-regulating EMT markers E-cadherin and claudin [34, 35]. Given the importance of EMT in the development of tumors and that the role of cell signaling pathways are unclear, further research is necessary to determine the mechanism of miR-939 and provide new targets for cancer treatment.

Although PMPs are accepted as an important means of intercellular communication, the mechanisms underlying PMPs internalization in recipient cells was poorly understood until Eric Boilard et al. found that 12(S)-hydroxyeicosatetranoic acid (12(S)-HETE) and sPLA $\mathrm{A}_{2}$-IIA activate the internalization by neutrophils [10]. SPLA $_{2}$-IIA is one of the oldest identified and studied enzymes. It is involved in various life-threatening diseases, such as asthma, diabetes, autoimmune disorders, rheumatoid arthritis, several neurological disorders [36], stable coronary heart disease [37] and various types of cancers (lung adenocarcinoma [38], gastric cancer [39], primary resected esophageal squamous cell carcinoma, and prostate cancer [40]. sPLA -IIA inhibition is considered an important therapeutic approach for different inflammatory diseases [41]. SPLA 2 -IIA is expressed by platelets in inflammation, as well as by cancer cells [40]. In our study, we found that $\mathrm{SPLA}_{2}$-IIA mediated the internalization of PMPs by ovarian cancer cells, which has not yet been reported. Given the effects of $\mathrm{sPLA}_{2}$-IIA in cancer cell internalization of PMPs, we believe that PLA $_{2}$-IIA could assist in tumor diagnosis and treatment in the near future.

Our study indicated that high levels of platelets could enhance the progression of EOC and lead to poor prognosis (Supplementary Figure 1). miR-939 might be an oncomiR and a new target for ovarian cancer treatment. Moreover, inhibiting the function of $\mathrm{SPLA}_{2}$-IIA may offer a new treatment for ovarian cancer. Ovarian cancer is difficult to diagnosis in the early stages. Therefore, new diagnostic markers are necessary. Perhaps in the near future, ovarian cancer diagnoses can be made using PMPs in the blood or urine. Therapiestargeting PMPs, miR-939 or $\mathrm{SPLA}_{2}$-IIA could significantly inhibit the progression of tumors and improve patient prognosis.

\section{MATERIALS AND METHODS}

\section{Clinical data}

From Nov 2008 to Jun 2013, 448 patients with an ovarian tumor who were undergoing treatment at Shanghai First Maternity and Infant Hospital were enrolled in this study. This group included 218 patients with benign epithelial ovarian tumors, 59 patients with borderline ovarian tumors with feature of lower malignant potential and good prognosis and 171 patients with EOC. This study was approved by the Institutional Review Board of the Shanghai First Maternity and Infant Hospital. Patients were excluded if they had incomplete preoperative laboratory data or if they underwent neoadjuvant therapy, suffered recurrent EOC or did not consent to the use of their medical records for research. For all EOC patients underwent debulking surgery and followed by 
chemotherapy based platinum. The median followup was at median 30 months (3-55 months). 123 patients obtained optimal surgery with less than $1 \mathrm{~cm}$ residue size tumor, the other 48 patients were suboptimal surgery.

\section{Clinical and laboratory data collection}

The predictive values studied included the area under receiver operating characteristic (ROC), sensitivity, specificity, positive predictive value (PPV), and negative predictive value (NPV). Optimal surgery was defined as surgery resulting in residual disease after debulking less than $1 \mathrm{~cm}$. Overall survival (OS) was assessed from diagnosis to the date of death from any cause or to the date of last contact.

\section{Microparticles}

\section{Platelet microparticles}

Platelets were derived from citrated blood of healthy human donors under an Institutional Review Board-approved protocol. Platelets were isolated after centrifugation of the blood $\left(1200 \mathrm{r}\right.$ for $30 \mathrm{~min}$ at $\left.21^{\circ} \mathrm{C}\right)$. Supernatant (platelet-rich plasma) was centrifuged at $2000 \mathrm{r}$ for $30 \mathrm{~min}$ at $21^{\circ} \mathrm{C}$. Pellet-containing platelets were resuspended in RPMI-1640 medium (HyClone, Logan, UT). Platelets were counted (Clinical Laboratory, Shanghai First Maternity and Infant Hospital, Shanghai) and adjusted to a density of $150 \times 10^{6}$ cells $/ \mathrm{ml}$ before they were supplemented with $1.5 \%$ Citrate-dextrose solution (ACD, Sigma, Sigma-Aldrich, St Louis, MO) and stimulated with thrombin $(1.0 \mathrm{u} / \mathrm{ml}$; Takeda Austria) for 1 hour. After centrifugation at $4000 \mathrm{r}$ for $10 \mathrm{~min}$ at $4^{\circ} \mathrm{C}$, the supernatant containing the PMPs was then centrifuged at $50,000 \times \mathrm{g}$ for $60 \mathrm{~min}$ at $4^{\circ} \mathrm{C}$. Pellets containing PMPs were resuspended in RPMI-1640 medium and quantified using the BCA method.

\section{Cell lines}

Human ovarian cancer cell line SKOV3 was purchased from the American Type Culture Collection at December 2013. The cell line was authenticated by STR test, and the cell line was tested in December 2015.

\section{Treatment of tumor cells with PMPs}

Cells were seeded in RPMI-1640 medium (HyClone, Logan, UT) with 10\% FBS (FBS, Gibco, USA) and $1 \%$ penicillin-streptomycin $(\mathrm{P} / \mathrm{S}, \mathrm{Gibco}$, USA) for 8 hours then started using empty medium for 4 hours. The medium was changed with $2 \%$ FBS 1640 . PMPs were added at a final concentration of approximately $100 \mu \mathrm{g} / \mathrm{ml}$.

\section{Cell proliferation analysis}

Approximately 3000 cells/well of SKOV3 cells were seeded in 96-well plates with difference concentrations of PMPs. Cultures were stained after 1, 2 , and 3 days. Culture absorbance was measured at 490 $\mathrm{nm}$ in a Thermo Scientific Multiskan (Thermo Fisher Scientific, USA) after incubating the cells with $20 \mu \mathrm{L}$ of a $5 \mathrm{mg} / \mathrm{ml} \mathrm{3-(4,5-dimethyl-2-thiazolyl)-2,5-diphenyl-2-}$ H-tetrazolium bromide (MTT, Sigma-Aldrich) solution at $37^{\circ} \mathrm{C}$ for 4 hours. The resulting MTT formosan was solubilized in dimethyl sulfoxide (DMSO).

\section{Migration assays}

For transwell migration assays, $1 \times 10^{4}$ cells were plated in the top chamber of a transwell containing a noncoated membrane (24-well insert; 8-mm pore size; BD Biosciences). Cells were plated in serum-free medium. Medium supplemented with $10 \%(\mathrm{v} / \mathrm{v})$ serum was used as a chemoattractant in the lower chamber. The experimental group was treated with $100 \mu \mathrm{g} / \mathrm{ml}$ PMPs and incubated for 16 hours at $37^{\circ} \mathrm{C}$ in a culture incubator with $5 \%(\mathrm{v} / \mathrm{v})$ $\mathrm{CO} 2$. Cells that migrated to the lower sides of the inserts were stained with Giemsa and counted.

\section{miRNA microarray}

Total miRNA expression of PMPs from platelets stimulated by thrombin or apoptosis were analyzed by Agilent's miRNA Complete Labeling and were identified on the Gene Expression Omnibus (http://ncbi.nlm.nih.gov/ geo, Design ID: 046064) website. Culture platelets in the empty medium for $48 \mathrm{~h}$ for natural apoptosis.

\section{Gene knockdown using small interfering RNA}

Small interfering RNAs for miR-939 (inhibitor) and sPLA $_{2}$-IIA (siRNA) were synthesized by Bioneer technology (Shanghai Genepharma Co., Ltd. Shanghai, CA). Cells were transfected with siRNA or inhibitor at a final concentration of $100 \mathrm{nM}$ using lipofectamine (Invitrogen), according to the manufacturer's suggested protocol. Gene knockdown was then verified by real-time quantitative polymerase chain reaction (qRT-PCR).

\section{Transient cell transfections}

miR-939 mimics, a negative control and miR939 inhibitor were purchased from GenePharma Co, Ltd (Shanghai, CA). These agents were transfected into SKOV3 cells using Lipofectamine_2000 reagent (Invitrogen) according to the manufacturer's instructions. Validation of miR-939 expression levels after transfection was performed using a PCR analysis. 


\section{RNA extraction and real-time quantitative PCR}

Total RNA including microRNAs was extracted from culture cells using Trizol reagents and treated with DNaseI. qRT-PCR were performed using the Two-Step PrimeScript miRNA cDNA Synthesis Kit (Takara, Dalian, China) using a ABI 7500 Real Time PCR system (Applied Biosystems, Foster City, CA, USA). Relative miRNA expression levels after normalization to U6 small nuclear RNA were calculated using 2 [(Ct of miR-939) (Ct of U6)]. qRT-PCR was performed by SYBR Kit (Qiagen, China) using a Light Cycler system. Relative mRNA expression levels were normalized to the geometric mean of $\beta$-actin to control for variability in expression levels and calculated.

\section{Western blotting}

The procedure used was referenced from Gillanders et al. [42] using a Bicinchoninic Acid (BCA) Protein Assay kit (Beyotime, China) to measure protein concentration. Primary antibodies (anti-E-cadherin, anti$\mathrm{N}$-cadherin, anti-vimentin, anti-slug and anti-snail) were purchased from Cell Signaling Technology, and Gapdh antibody (Sigma-Aldrich) was used as a normalization control. The secondary antibody was a horseradish peroxidase-conjugated antibody (Sigma-Aldrich) that was incubated for 1 hour at room temperature. Enhanced chemiluminescence (ECL) was used to visualize immunocomplexes following the manufacturer's protocol.

\section{Confocal microscopy}

PMPs were stained with D384, a phospholipid membrane dye (red stain), and SytoRNA Select, an RNAselect stain (green stain). Then, $100 \mu \mathrm{g} / \mathrm{ml}$ PMPs was incubated with unstained naive recipient cells for 12 hours.

\section{Statistical analysis}

SPSS ver. 20.0 (SPSS Inc., Chicago, IL, USA) and GraphPad Prism Software (version) were used for statistical analyses. Data from the different groups were compared using a chi-square test or Fisher's exact test. OS was calculated using a Kaplan-Meier method to determine the univariate relationship of thrombocytosis. ROC curves were plotted and the areas under the curve(AUC) were calculated for comparison. The ROC curves were generated by plotting the relationship of the true positivity (sensitivity) and the false positivity (1-specificity) at various cut off points of the tests. A p-value $<0.05$ was considered statistically significant.

\section{CONFLICTS OF INTEREST}

No potential conflicts of interest were disclosed.

\section{FUNDING}

This study was supported by grants from the National Science Foundation of China (81372787, 81072136), Shanghai Municipal Bureau of Health (20134033), Shanghai Health System joint research project (2013ZYJB0201), Shanghai Scientific and Technology commission (17411968100).

\section{REFERENCES}

1. Siegel RL, Miller KD, Jemal A. Cancer statistics, 2015. CA Cancer J Clin. 2015; 65:5-29.

2. Davis AN, Afshar-Kharghan V, Sood AK. Platelet effects on ovarian cancer. Semin Oncol. 2014; 41:378-384.

3. Morgan MA, Iyengar TD, Napiorkowski BE, Rubin SC, Mikuta JJ. The clinical course of deep vein thrombosis in patients with gynecologic cancer. Gynecol Oncol. 2002; 84:67-71.

4. Stone RL, Nick AM, McNeish IA, Balkwill F, Han HD, Bottsford-Miller J, Rupairmoole R, Armaiz-Pena GN, Pecot CV, Coward J, Deavers MT, Vasquez HG, Urbauer D, et al. Paraneoplastic thrombocytosis in ovarian cancer. N Engl J Med. 2012; 366:610-618.

5. Cetinkunar S, Guzel H, Emre Gokce I, Erdem H, Gulkan S, Aktimur R, Kucuk B, Imamoglu I, Kargici H. High levels of platelet/lymphocyte ratio are associated with metastatic gastric cancer. J BUON. 2015; 20:78-83.

6. Radomski MW, Jenkins DC, Holmes L, Moncada S. Human colorectal adenocarcinoma cells: differential nitric oxide synthesis determines their ability to aggregate platelets. Cancer Res. 1991; 51:6073-6078.

7. Gay LJ, Felding-Habermann B. Contribution of platelets to tumour metastasis. Nat Rev Cancer. 2011; 11:123-134.

8. Borsig L. The role of platelet activation in tumor metastasis. Expert Rev Anticancer Ther. 2008; 8:1247-1255.

9. Ho-Tin-Noe B, Goerge T, Wagner DD. Platelets: guardians of tumor vasculature. Cancer Res. 2009; 69:5623-5626.

10. Liu Y, Goswami RK, Liu C, Sinha SC. Chemically programmed bispecific antibody targeting legumain protease and alphavbeta3 integrin mediates strong antitumor effects. Mol Pharm. 2015; 12:2544-2550.

11. Duchez AC, Boudreau LH, Naika GS, Bollinger J, Belleannée C, Cloutier N, Laffont B, Mendoza-Villarroel RE, Lévesque T, Rollet-Labelle E, Rousseau M, Allaeys I, Tremblay JJ, et al. Platelet microparticles are internalized in neutrophils via the concerted activity of 12-lipoxygenase and secreted phospholipase A2-IIA. Proc Natl Acad Sci U S A. 2015; 112:E3564-E3573.

12. Burnouf T, Goubran HA, Chou ML, Devos D, Radosevic M. Platelet microparticles: detection and assessment of their paradoxical functional roles in disease and regenerative medicine. Blood Rev. 2014; 28:155-166. 
13. Allensworth SK, Langstraat CL, Martin JR, Lemens MA, McGree ME, Weaver AL, Dowdy SC, Podratz KC, Bakkum-Gamez JN. Evaluating the prognostic significance of preoperative thrombocytosis in epithelial ovarian cancer. Gynecol Oncol. 2013; 130:499-504.

14. Asher V, Lee J, Innamaa A, Bali A. Preoperative platelet lymphocyte ratio as an independent prognostic marker in ovarian cancer. Clin Transl Oncol. 2011; 13:499-503.

15. Atacag T. Diagnostic value of thrombocytosis and high CA 125 level in women with adnexal masses. Eur J Gynaecol Oncol. 2012; 33:517-520.

16. Qiu J, Yu Y, Fu Y, Ye F, Xie X, Lu W. Preoperative plasma fibrinogen, platelet count and prognosis in epithelial ovarian cancer. J Obstet Gynaecol Res. 2012; 38:651-657.

17. Lee M, Kim SW, Nam EJ, Yim GW, Kim S, Kim YT. The impact of pretreatment thrombocytosis and persistent thrombocytosis after adjuvant chemotherapy in patients with advanced epithelial ovarian cancer. Gynecol Oncol. 2011; 122:238-241.

18. Gungor T, Kanat-Pektas M, Sucak A, Mollamahmutoglu L. The role of thrombocytosis in prognostic evaluation of epithelial ovarian tumors. Arch Gynecol Obstet. 2009; 279:53-56.

19. Li A. The prognostic significance of thrombocytosis in epithelial ovarian carcinoma. Gynecol Oncol. 2004; 92:211-214.

20. Green D, Hull RD, Brant R, Pineo GF. Lower mortality in cancer patients treated with low-molecular-weight versus standard heparin. Lancet. 1992; 339:1476.

21. Siragusa S, Cosmi B, Piovella F, Hirsh J, Ginsberg JS. Lowmolecular-weight heparins and unfractionated heparin in the treatment of patients with acute venous thromboembolism: results of a meta-analysis. Am J Med. 1996; 100:269-277.

22. Hettiarachchi RJ, Smorenburg SM, Ginsberg J, Levine M, Prins MH, Büller HR. Do heparins do more than just treat thrombosis? The influence of heparins on cancer spread. Thromb Haemost. 1999; 82:947-952.

23. Cosgrove RH, Zacharski LR, Racine E, Andersen JC. Improved cancer mortality with low-molecular-weight heparin treatment: a review of the evidence. Semin Thromb Hemost. 2002; 28:79-87.

24. Kakkar AK, Williamson RC. Antithrombotic therapy in cancer. BMJ. 1999; 318:1571-1572.

25. Falanga A, Tartari CJ, Marchetti M. Microparticles in tumor progression. Thromb Res. 2012; 129:S132-S136.

26. Campello E, Spiezia L, Radu CM, Bulato C, Castelli M, Gavasso S, Simioni P. Endothelial, platelet, and tissue factor-bearing microparticles in cancer patients with and without venous thromboembolism. Thromb Res. 2011; 127:473-477.

27. Dashevsky O, Varon D, Brill A. Platelet-derived microparticles promote invasiveness of prostate cancer cells via upregulation of MMP-2 production. Int J Cancer. 2009; 124:1773-1777.
28. Hayon Y, Dashevsky O, Shai E, Varon D, Leker RR. Platelet microparticles promote neural stem cell proliferation, survival and differentiation. J Mol Neurosci. 2012 47:659-665.

29. Rani S, Gately K, Crown J, O’Byrne K, O’Driscoll L. Global analysis of serum microRNAs as potential biomarkers for lung adenocarcinoma. Cancer Biol Ther. 2013; 14:1104-1112.

30. Nymark P, Guled M, Borze I, Faisal A, Lahti L, Salmenkivi $\mathrm{K}$, Kettunen E, Anttila S, Knuutila S. Integrative analysis of microRNA, mRNA and aCGH data reveals asbestosand histology-related changes in lung cancer. Genes Chromosomes Cancer. 2011; 50:585-597.

31. Scaruffi P, Stigliani S, Moretti S, Coco S, De Vecchi C, Valdora F, Garaventa A, Bonassi S, Tonini GP. Transcribedultra conserved region expression is associated with outcome in high-risk neuroblastoma. BMC Cancer. 2009; 9:441.

32. Ma R, Wang C, Wang J, Wang D, Xu J. miRNA-mRNA interaction network in non-small cell lung cancer. Interdiscip Sci. 2016; 8:209-219.

33. Ying X, Li-ya Q, Feng Z, Yin W, Ji-hong L. MiR-939 promotes the proliferation of human ovarian cancer cells by repressing APC2 expression. Biomed Pharmacother. 2015; 71:64-69.

34. Che J, Yang Y, Xiao J, Zhao P, Yan B, Dong S, Cao B. Decreased expression of claudin-3 is associated with a poor prognosis and EMT in completely resected squamous cell lung carcinoma. Tumour Biol. 2015; 36:6559-6568.

35. Zeisberg M, Neilson EG. Biomarkers for epithelialmesenchymal transitions. J Clin Invest. 2009; 119:1429-1437.

36. Yagami T, Yamamoto Y, Koma H. The role of secretory phospholipase $\mathrm{A}(2)$ in the central nervous system and neurological diseases. Mol Neurobiol. 2014; 49:863-876.

37. Shuvalova YA, Khasanova ZB, Kaminnaya VI, Samoilova EV, Korotaeva AA, Rubanovich AV, Kaminnyi AI. The association of PLA2G2A single nucleotide polymorphisms with type IIa secretory phospholipase A2 level but not its activity in patients with stable coronary heart disease. Gene. 2015; 564:29-34.

38. Kupert E, Anderson M, Liu Y, Succop P, Levin L, Wang J, Wikenheiser-brokamp K, Chen P, Pinney SM, Macdonald T, Dong Z, Starnes S, Lu S. Plasma secretory phospholipase A2-IIa as a potential biomarker for lung cancer in patients with solitary pulmonary nodules. BMC Cancer. 2011; $11: 513$.

39. Xing XF, Li H, Zhong XY, Zhang LH, Wang XH, Liu YQ, Jia SQ, Shi T, Niu ZJ, Peng Y, Du H, Zhang GG, Hu Y, et al. Phospholipase A2 group IIA expression correlates with prolonged survival in gastric cancer. Histopathology. 2011; 59:198-206.

40. Dong Z, Liu Y, Scott KF, Levin L, Gaitonde K, Bracken RB, Burke B, Zhai QJ, Wang J, Oleksowicz L, Lu S. 
Secretory phospholipase A2-IIa is involved in prostate cancer progression and may potentially serve as a biomarker for prostate cancer. Carcinogenesis. 2010; 31:1948-1955.

41. Farooqui AA, Ong WY, Horrocks LA. Inhibitors of brain phospholipase A2 activity: their neuropharmacological effects and therapeutic importance for the treatment of neurologic disorders. Pharmacol Rev. 2006; 58:591-620.
42. Tiriveedhi V, Tucker N, Herndon J, Li L, Sturmoski M, Ellis M, Ma C, Naughton M, Lockhart AC, Gao F, Fleming T, Goedegebuure P, Mohanakumar T, Gillanders WE. Safety and preliminary evidence of biologic efficacy of a mammaglobin-a DNA vaccine in patients with stable metastatic breast cancer. Clin Cancer Res. 2014; 20:5964-5975. 